\title{
REFLEXÕES SOBRE A INTELIGÊNCIA ARTIFICIAL NA AGENDA 2030 PARA O DESENVOLVIMENTO SUSTENTÁVEL
}

\author{
REFLECTIONS ON ARTIFICIAL INTELLIGENCE IN THE 2030 \\ AGENDA FOR SUSTAINABLE DEVELOPMENT
}

\section{Sthéfano Bruno Santos Divino ${ }^{1}$}

\section{RESUMO}

O presente artigo tem como problema de pesquisa 0 seguinte questionamento: como a Inteligência Artificial (IA) pode contribuir para o cumprimento das metas da Agenda 2030 para o Desenvolvimento sustentável? A primeira seção se incumbe da demonstração das possíveis utilizações da IA para o efetivo atingimento dos Objetivos de Desenvolvimento Sustentável (ODS). Neste momento, destacam-se os aspectos positivos e os aspectos negativos na utilização da IA. Na segunda seção, realiza-se um estudo sobre os atos normativos de alguns países sobre as possíveis implicações negativas e regulamentais da IA para com a sociedade. Para tanto, utiliza-se os métodos dedutivo e de pesquisa integrada. Ao final, demonstra-se que a IA apresenta-se como importante fator da equação posta pela Agenda 2030, desde que devidamente observada e regulada.

PALAVRAS-CHAVE: Agenda 2030; Inteligência Artificial; Desenvolvimento Sustentável.

\section{ABSTRACT}

This article has as a research problem the following question: how can Artificial Intelligence (AI) contribute to meeting the goals of the 2030 Agenda for Sustainable Development? The first section is responsible for demonstrating the possible uses of AI for the effective achievement of the Sustainable Development Goals (SDGs). At this moment, the positive and negative aspects in the use of AI are highlighted. In the second section, a

\footnotetext{
1 Doutorando e Mestre em Direito Privado pela Pontifícia Universidade Católica de Minas Gerais (2019) - PUC-MG. Bacharel em Direito pelo Centro Universitário de Lavras - Unilavras (2017). Professor substituto de Direito Privado na Universidade Federal de Lavras. Advogado. Lavras, Minas Gerais, Brasil. sthefanoadv@hotmail.com
} 
SANTOS DIVINO, Sthéfano Bruno. Reflexões sobre a inteligência artificial na agenda 2030 para o desenvolvimento sustentável. Revista Eletrônica Direito e Política, Programa de Pós-Graduação Stricto Sensu em Ciência Jurídica da UNIVALI, Itajaí, v.16, n.1, $1^{0}$ quadrimestre de 2021. Disponível em: www.univali.br/direitoepolitica - ISSN 1980-7791.

study is carried out on the normative acts of some countries on the possible negative and regulatory implications of AI for society. For this, the deductive and integrated research methods are used. In the end, it is shown that AI presents itself as an important factor in the equation posed by Agenda 2030, as long as it is properly observed and regulated.

Keywords: Agenda 2030; Artificial intelligence; Sustainable development.

\section{INTRODUÇÃO}

O desenvolvimento da Inteligência Artificial tem se intensificado nos últimos anos. Ressalta-se o surgimento de técnicas como machine learning e deep learning para o uso contínuo do aprendizado de máquinas. Essas aplicações apresentam na contemporaneidade utilidades e praticidades se implementadas objetivando a tendência de evolução e desenvolvimento sustentável. Vários são os usos da IA, o que ganha relevância, de maneira nova, que se torna fonte direta e contínua de aproveitamento no presente trabalho é aquele destinado à satisfação da Agenda 2030.

O presente trabalho tem como problema de pesquisa o seguinte questionamento: como a Inteligência Artificial (IA) pode contribuir para o cumprimento das metas da Agenda 2030 para o Desenvolvimento sustentável?

Tomam-se caminhos simplificados, mas objetivos, com a pretensão da satisfação dessa problemática. A primeira seção trata de forma concreta e específica qual a relação entre Inteligência Artificial e os Objetivos de Desenvolvimento Sustentável (ODS) propostos pela Agenda 2030. Abordase, através da demonstração de relatórios da UNESCO, a correlação entre os ODS's direcionados à água, saúde, agricultura e educação.

Demonstra-se no decorrer argumentativo as possíveis implicações positivas e negativas do uso da IA na sociedade contemporânea. Desta forma podese acompanhar um raciocínio aplicável aos setores governamentais e 
SANTOS DIVINO, Sthéfano Bruno. Reflexões sobre a inteligência artificial na agenda 2030 para o desenvolvimento sustentável. Revista Eletrônica Direito e Política, Programa de Pós-Graduação Stricto Sensu em Ciência Jurídica da UNIVALI, Itajaí, v.16, n.1, $1^{0}$ quadrimestre de 2021. Disponível em: www.univali.br/direitoepolitica - ISSN 1980-7791.

jurídicos. O que se pretende é enunciar os possíveis riscos e efeitos colaterais desse tipo de tecnologia. Assim, a segunda seção é responsável pela abordagem das regulamentações e eventuais políticas governamentais destinadas a diminuição ou erradicação desses efeitos aparentemente nocivos à sociedade. Países como China, Reino Unido e Canadá são utilizados como objeto de pesquisa em virtude de sua ampla influência nesse cenário.

Ao final, verifica-se que os efeitos positivos trazidos pela adoção das tecnologias envoltas pela inteligência artificial podem auxiliar ao efetivo cumprimento da Agenda 2030. Lado outro, as consequências pormenorizadas, principalmente ligadas ao aumento da possível desigualdade interna e externa já estão sendo tratadas e discutidas pelos responsáveis pelo desenvolvimento da IA como problemas eventualmente a serem enfrentados. Situações éticas e morais já podem ser detectadas e discutidas em cartilhas e normativos para evitar ou diminuir os possíveis riscos da adoção da IA para o desenvolvimento tecnológico sustentável. Para chegar ao presente raciocínio, utiliza-se os métodos dedutivo e de pesquisa integrada.

\section{A RELAÇÃo ENTRE INTELIGÊNCIA ARTIFICIAL E A AGENDA 2030} PARA O CUMPRIMENTO DOS OBJETIVOS DE DESENVOLVIMENTO SUSTENTÁVEL

O campo das ciências voltado para o estudo da Inteligência Artificial (IA) tem crescido nos últimos anos. Apesar dos incontáveis estudos nas últimas décadas sobre o aspecto conceitual ${ }^{2}$ ou ontológico ${ }^{3}$ de uma Inteligência

\footnotetext{
2 "It is the science and engineering of making intelligent machines, especially intelligent computer programs. It is related to the similar task of using computers to understand human intelligence, but AI does not have to confine itself to methods that are biologically observable". MCCARTHY, J. What is artificial intelligence? Stanford University, p. 2-15, 2007

3 "I find it useful to distinguish what I will call "strong" AI from "weak" or "cautious" AI (Artificial Intelligence). According to weak AI, the principal value of the computer in the study of the mind is
} 
SANTOS DIVINO, Sthéfano Bruno. Reflexões sobre a inteligência artificial na agenda 2030 para o desenvolvimento sustentável. Revista Eletrônica Direito e Política, Programa de Pós-Graduação Stricto Sensu em Ciência Jurídica da UNIVALI, Itajaí, v.16, n.1, 10 quadrimestre de 2021. Disponível em: www.univali.br/direitoepolitica - ISSN 1980-7791.

Artificial, o presente artigo pretende, de forma a não os ignorar, mas também não abordar essas temáticas com a profundidade necessária, demonstrar objetivamente as possibilidades fáticas de como a IA pode contribuir para a Agenda 2030.

Para compreensão da funcionalidade dos sistemas de IA's, algumas abordagens prévias devem ser tecidas. Primeiramente, o campo dos entes inteligentes artificialmente pode ser bipartido. De um lado tem-se a abordagem baseada em regras, onde os pesquisadores do campo, tentam ensinar os computadores a pensar codificando uma série de regras lógicas no sistema. De outro, o campo das redes neurais, também denominada aprendizado profundo, adota uma abordagem diferente, nas quais os pesquisadores e cientistas tentam reconstruir o próprio cérebro humano. Tal abordagem tende a simular a arquitetura do cérebro humano para construir camadas de neurônios artificiais capazes de receber e transmitir informações em uma estrutura aparentemente semelhante às redes de neurônios biológicos. ${ }^{4}$

Existe uma tríade correlacional para o aprendizado comum funcionar da forma esperada. O primeiro deles é o poder computacional. Expresso através da dualidade hardware-software, esse conjunto será o responsável pela execução do segundo elemento da tríade, o algoritmo. Este é um código ou uma função desenvolvida pelo programador para executar o programa de aprendizado profundo. Contudo, o terceiro pilar nessa seara do deep learning apresenta uma maior importância: dados. Os dados possuem a capacidade de treinar o programa para reconhecimento de

that it gives us a very powerful tool. For example, it enables us to formulate and test hypotheses in a more rigorous and precise fashion. But according to strong AI, the computer is not merely a tool in the study of the mind; rather, the appropriately programmed computer really is a mind, in the sense that computers given the right programs can be literally said to understand and have other cognitive states. In strong AI, because the programmed computer has cognitive states, the programs are not mere tools that enable us to test psychological explanations; rather, the programs are themselves the explanation". SEARLE, J. Minds, brains, and programs. Behavioral and Brain Sciences. Vol. 3, n. 3. 1980, p. 417-424.

${ }^{4}$ LEE, Kai-Fu. Inteligência Artificial. Rio de Janeiro: Globo Livros, 2019, p. 20-21. 
SANTOS DIVINO, Sthéfano Bruno. Reflexões sobre a inteligência artificial na agenda 2030 para o desenvolvimento sustentável. Revista Eletrônica Direito e Política, Programa de Pós-Graduação Stricto Sensu em Ciência Jurídica da UNIVALI, Itajaí, v.16, n.1, $1^{0}$ quadrimestre de 2021. Disponível em: www.univali.br/direitoepolitica - ISSN 1980-7791.

padrões. Esses padrões podem ser executados através do poder computacional em alta velocidade para o próprio algoritmo aprendê-los e superá-los de uma forma incomparável ${ }^{5}$ aos sentidos humanos.

Dessa forma, a conexão entre essa tríade pode ser expressa da seguinte forma:

Figura $1^{6}$

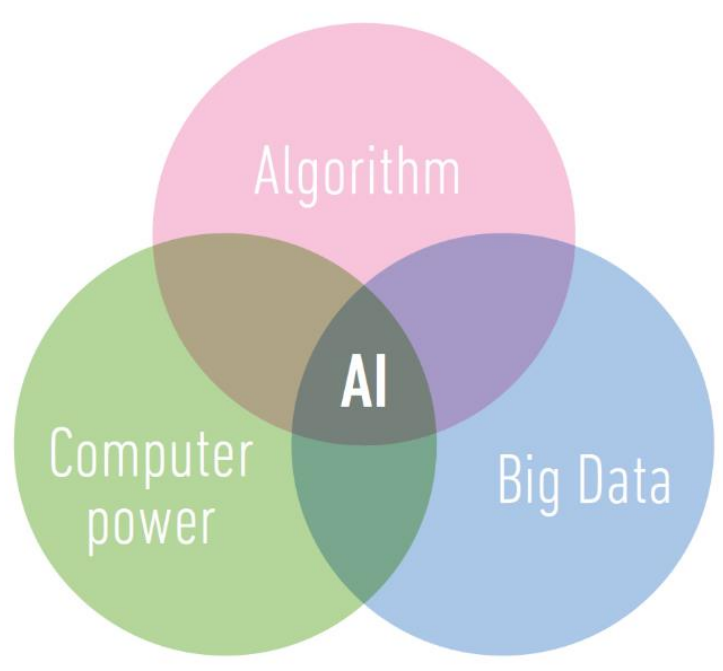

Esse ecossistema revela uma premissa lógica: quanto maior o número de informação, maior a capacidade de o deep learning fornecer respostas aos problemas inicialmente propostos. Esse processo exige muito trabalho e adaptação que às vezes poderiam demorar meses ou até anos se executados pelo cérebro humano. Ainda que especialistas atuem na área, quando comparados a incontável razão produtiva de matéria-prima fornecida pelo Big Data, o aprendizado profundo inaugura uma situação em que "estamos passando da era da especialização para a era dos dados". ${ }^{7}$ Esse salto, contudo, pode ser benéfico se aplicado como ferramentas

\footnotetext{
5 Para Andrew $\mathrm{Ng}$, um dos principais responsáveis pelo deep learning, a IA pode ser comparada ao aproveitamento da energia idealizado por Thomas Edison. LYNCH, Shana. Andrew Ng: Why AI Is the New Electricity. Stanford Business. 2017. https://www.gsb.stanford.edu/insights/andrew-ng-whyai-new-electricity

${ }^{6}$ UNESCO, Artificial intelligence for sustainable development: challenges and opportunities for UNESCO's science and engineering programmes. 2019, p. 11.

7 LEE, Kai-Fu. Inteligência Artificial. Rio de Janeiro: Globo Livros, 2019, p. 74.
} 
SANTOS DIVINO, Sthéfano Bruno. Reflexões sobre a inteligência artificial na agenda 2030 para o desenvolvimento sustentável. Revista Eletrônica Direito e Política, Programa de Pós-Graduação Stricto Sensu em Ciência Jurídica da UNIVALI, Itajaí, v.16, n.1, $1^{0}$ quadrimestre de 2021. Disponível em: www.univali.br/direitoepolitica - ISSN 1980-7791.

conexas que coletam dados do mundo real e destinam de forma eficaz o que fazer com eles. E é nesse ponto que abordaremos os ODS.

\subsection{Inteligência Artificial e Gerenciamento de Água: ODS's 6, 12 e} 14

Os Objetivos de Desenvolvimento Sustentável número 6, 12 e 14 se referem, respectivamente: a garantia de disponibilidade e gestão sustentável da água e saneamento para todos; garantia de padrões de consumo e produção sustentáveis; e conservar e usar de forma sustentável os oceanos, mares e recursos marinhos. As funcionalidades do sistema de IA permitem a adoção de técnicas e atividades para formar uma base sólida para os esforços pretendidos.

Com a utilização dos sistemas da Internet das Coisas (IoT - Internet of Things), a Fracta, startup sediada em Redwood City na Califórnia, elaborou um sistema que objetiva auxiliar as concessionárias de água a economizar dinheiro e recursos, priorizando a substituição de canos de água e canos de distribuição nas cidades, a partir da qual canos menores se ramificam em prédios. $^{8}$

Stenstedt diz que as concessionárias já estão procurando analisar o maior número possível de variáveis que entram no ciclo de vida de um determinado tubo, mas "à medida que o número de variáveis relativas aumenta, a única maneira de abordar isso é através do machine learning." Dessa forma, a Fracta analisa e correlaciona os dados ao tipo de solo em que os canos repousam, a topografia e os registros meteorológicos e, em seguida, aplica o aprendizado de máquina para encontrar padrões, em toda uma cidade ou região, que oferecem indícios sobre quais encanamentos

\footnotetext{
8 O'CONNOR, Marry Catherine. How AI Could Smarten Up Our Water System. Medium. 2017.
} https://medium.com/s/ai-for-good/how-ai-could-smarten-up-our-water-system-f965b87f355a 
SANTOS DIVINO, Sthéfano Bruno. Reflexões sobre a inteligência artificial na agenda 2030 para o desenvolvimento sustentável. Revista Eletrônica Direito e Política, Programa de Pós-Graduação Stricto Sensu em Ciência Jurídica da UNIVALI, Itajaí, v.16, n.1, $1^{0}$ quadrimestre de 2021. Disponível em: www.univali.br/direitoepolitica - ISSN 1980-7791.

estão sob maior risco de vazamentos ou falha. A empresa está atualmente trabalhando com duas concessionárias de água na Bay Area para provar que sua abordagem funciona. ${ }^{9}$

A empresa Valor Water Analytics, localizada em São Francisco, desenvolveu algoritmos de machine learning para detectar imprecisões ou anomalias nos dados do medidor de seus clientes. Dessa forma, tornam-se capaz de localizar onde a água consumida pode ter sido não faturada, conduta essa que custa bilhões aos cofres públicos. Mas a Valor também fornece aos utilitários as ferramentas que eles podem usar para trabalhar com os clientes para reduzir o uso da água e identificar vazamentos. ${ }^{10}$

Na Sérvia, por exemplo, o Centro de Água para o Desenvolvimento Sustentável e a Adaptação às Mudanças Climáticas, que opera sob a autoridade da UNESCO, usa IA e modelagem estatística para controlar a qualidade dos dados obtidos para o monitoramento estrutural e ambiental das condições climáticas. ${ }^{11}$

O aplicativo G-WADI da UNESCO (Water and Development Information for Arid Lands - a Global Network) usa um algoritmo de rede neural artificial (RNA) para estimar a precipitação em tempo real em todo o mundo. Esse produto é chamado de Precipitation Estimation from Remotely Sensed Information using Artificial Neural Networks - Cloud Classification System (G-WADI PERSIANN-CCS6). Através do G-WADI é possível realizar o planejamento e o gerenciamento de emergência de riscos hidrológicos, tais como inundações, secas e eventos climáticos extremos. O sistema está disponível através do aplicativo móvel iRain, objetivando o envolvimento do

\footnotetext{
9 O'CONNOR, Marry Catherine. How AI Could Smarten Up Our Water System. Medium. 2017. https://medium.com/s/ai-for-good/how-ai-could-smarten-up-our-water-system-f965b87f355a 10 O'CONNOR, Marry Catherine. How AI Could Smarten Up Our Water System. Medium. 2017. https://medium.com/s/ai-for-good/how-ai-could-smarten-up-our-water-system-f965b87f355a

11 UNESCO. Artificial intelligence for sustainable development: challenges and opportunities for UNESCO's science and engineering programmes. 2019, p. 19.
} 
SANTOS DIVINO, Sthéfano Bruno. Reflexões sobre a inteligência artificial na agenda 2030 para o desenvolvimento sustentável. Revista Eletrônica Direito e Política, Programa de Pós-Graduação Stricto Sensu em Ciência Jurídica da UNIVALI, Itajaí, v.16, n.1, $1^{0}$ quadrimestre de 2021. Disponível em: www.univali.br/direitoepolitica - ISSN 1980-7791.

cidadão na coleta de dados locais para o monitoramento global da precipitação ${ }^{12}{ }^{13}$

Uma vantagem existente com a implementação dos sistemas que utilizam a IoT é a relação de custos. A princípio não requer nenhuma infraestrutura física de grande porte e pré-existente. Ela pode ser implantada na maioria das áreas remotas. A vasta gama de sensores que podem ser conectados à rede suporta inúmeras aplicações científicas diferentes, incluindo as disposições já expressas pela Fracta ou Valor Water Analytics. Dessa forma, como reflexo, consegue-se cumprir de forma reflexa o ODS 3 (garantia de uma vida saudável e promoção do bem-estar para todos em todas as idades).

Mas não é apenas nesse setor que a utilização da IA se destaca para a funcionalidade dos primeiros ajustes e otimizações do cumprimento dos ODS's. isso abre uma oportunidade real para extensão e empreendedorismo em massa das inovações tecnológicas voltadas ao desenvolvimento sustentável. E outro campo atingido é o da agricultura.

\subsection{Inteligência Artificial e Agricultura: ODS's 2, 9 e 10}

O entomologista David Hughes e o epidemiologista Marcel Salathé, ambos do Instituto Federal Suíço de Tecnologia (EPFL - Swiss Federal Institute of Technology) em Lausanne, Suíça, conjuntamente com a Universidade Estadual de Penn, são responsáveis pela elaboração do projeto PlantVillage.

\footnotetext{
12 Rasp et al. Desenvolveram um programa de Inteligência Artificial baseado no aprendizado profundo capaz de melhorar as deteç̧ões e previsões climáticas quando analisadas através de simulações de curto prazo. Para mais, ver em: RASP, Stephan; PRITCHARD, Michael S; GENTINE, Pierre. Deep learning to represent subgrid processes in climate models. PNAS. Vol. 115, n. 39, p. 9684-9689. https://doi.org/10.1073/pnas.1810286115. https://www.pnas.org/content/pnas/115/39/9684.full.pdf 13 RASP, Stephan; PRITCHARD, Michael S; GENTINE, Pierre. Deep learning to represent subgrid processes in climate models. PNAS. Vol. 115, n. 39, p. 9684-9689. https://doi.org/10.1073/pnas.1810286115. https://www.pnas.org/content/pnas/115/39/9684.full.pdf.
} 
SANTOS DIVINO, Sthéfano Bruno. Reflexões sobre a inteligência artificial na agenda 2030 para o desenvolvimento sustentável. Revista Eletrônica Direito e Política, Programa de Pós-Graduação Stricto Sensu em Ciência Jurídica da UNIVALI, Itajaí, v.16, n.1, $1^{0}$ quadrimestre de 2021. Disponível em: www.univali.br/direitoepolitica - ISSN 1980-7791.

O principal objetivo é fazer com que um agricultor consiga diagnosticar doenças em sua cultura utilizando apenas um aplicativo de smartphone. ${ }^{14}$ Os desenvolvedores justificam a criação do projeto tendo em vista que as doenças de plantas que atingem certas comunidades podem ser desastrosas, vez que atingem os meios de subsistência dos referidos. ${ }^{15}$

O aplicativo funciona com uma base ligada a um banco de dados com aproximadamente 150.000 (cento e cinquenta mil) fotografias de plantas com enfermidades. Pretende-se que tal número nos próximos anos chegue a três milhões. No contexto contemporâneo, as análises são voltadas para o que a equipe acredite ser as dez doenças mais importantes que afetam as trinta culturas mais importantes.

Os desenvolvedores utilizam uma IA integrante de uma rede neural de deep learning capaz de identificar com 98,21\% de chance de acerto qual planta e qual o tipo de doença que está sendo analisada.

Neural networks provide a mapping between an input, such as an image of a diseased plant, to an output, such as a crop-disease pair. Deep neural networks recently have been applied successfully in many diverse domains. These networks are trained by tuning the network parameters in such a way that the mapping improves during the training process. ${ }^{16}$

Com a tecnologia em pauta, verifica-se a possibilidade de dados serem utilizados para identificar patologias e fazer melhores predições sobre quando e onde elas aparecerão. ${ }^{17} \mathrm{O}$ impacto do PlantVillage pode ser

${ }^{14}$ BREWSTER, S. Wilting shrubs? Diagnose plant diseases with an app. MIT Technology Review. 2016. https://www.technologyreview.com/s/602792/wilting-shrubs-diagnose-plant-diseases-withan-app/

15 CHUCK, Gill. Artificial intelligence could help farmers diagnose crop diseases. 2017. http://news.psu.edu/story/429727/2016/10/04/research/artificial-intelligence-couldhelp-farmersdiagnose-crop-diseases

${ }_{16}$ CHUCK, Gill. Artificial intelligence could help farmers diagnose crop diseases. 2017. http://news.psu.edu/story/429727/2016/10/04/research/artificial-intelligence-couldhelp-farmersdiagnose-crop-diseases

17 LOHR, S. From agriculture to art - the AI wave sweeps in. New York Times, Business Day. 2019. https://www.nytimes.com/2018/10/21/business/from-agriculture-to-art-the-ai-wave-sweepsin.html 
SANTOS DIVINO, Sthéfano Bruno. Reflexões sobre a inteligência artificial na agenda 2030 para o desenvolvimento sustentável. Revista Eletrônica Direito e Política, Programa de Pós-Graduação Stricto Sensu em Ciência Jurídica da UNIVALI, Itajaí, v.16, n.1, $1^{0}$ quadrimestre de 2021. Disponível em: www.univali.br/direitoepolitica - ISSN 1980-7791.

verificado conforme os números extraídos dos relatórios dos plantios. Segundo Hughes, plantações de mandioca alimentam cerca de 600 milhões de pessoas. Além disso, apenas na Nigéria ${ }^{18}$ a circulação econômica desse plantio gira em torno de 2 bilhões de dólares. A utilização do projeto pode reduzir a porcentagem de $40 \%$ de perda da plantação em caso de epidemia no cultivo. ${ }^{19}$

Outro exemplo de tecnologia envolvendo IA no setor agrícola é o sistema Sequoia, desenvolvido pela Parrot, uma empresa francesa. O Sequoia é um sensor multiespectral conectado a um drone que combina uma câmera com um sensor solar GPS (raios ultravioleta e infravermelho) para analisar a vitalidade da colheita capturando a quantidade de luz que as plantas absorvem e refletem. ${ }^{20}$

Esses tipos de projetos que estão correlacionados possuem uma capacidade de alto impacto social. Nos principais países de baixa renda, como o é os em análise, existe uma carência de estudos e insumos governamentais voltados para as ciências agrícolas. A utilização das tecnologias da informação e da comunicação, associadas aos dispositivos interconectados da IoT podem ser capazes de ajudar a quebrar o ciclo de pobreza, vez que os responsáveis pelo cultivo terão as informações necessárias na palma de suas mãos de forma imediata e com custos extremamente reduzidos, já que, por exemplo, no caso do PlantVillage, uma vez baixado o aplicativo, dispensa-se a conexão com a internet para o seu funcionamento. Assim, esse tipo de projeto demonstra potencial de aumentar a produtividade dos

\footnotetext{
18 In Nigeria, AI is being employed to help farmers sell their produce and buy services via a bot platform that relies on SMS and other channels such as USSD, Slack, etc. WWWF. Artificial Intelligence: the Road Ahead in Low and Middle-Income Countries. World Wide Web Foundation. 2017. https://webfoundation.org/docs/2017/07/AI Report WF.pdf

19 GORALSKI, Margareth A.; TAN, Tay Keong. Artificial intelligence and sustainable development. The International Journal of Management Education. Vol. 18, 2020, p. 5.

20 IM'NOVATION. https://www.imnovation-hub.com/society/types-of-smart-sensors-applied-toagriculture/\#0

UNESCO, Artificial intelligence for sustainable development: challenges and opportunities for UNESCO's science and engineering programmes. 2019, p 16.
} 
SANTOS DIVINO, Sthéfano Bruno. Reflexões sobre a inteligência artificial na agenda 2030 para o desenvolvimento sustentável. Revista Eletrônica Direito e Política, Programa de Pós-Graduação Stricto Sensu em Ciência Jurídica da UNIVALI, Itajaí, v.16, n.1, $1^{0}$ quadrimestre de 2021. Disponível em: www.univali.br/direitoepolitica - ISSN 1980-7791.

sistemas agrícolas, melhorar a produção agrícola e melhorar a produção de alimentos.

Com isso, os ODS's 2 (acabar com a fome, alcançar a segurança alimentar e melhorar a nutrição e promover a agricultura sustentável), 9 (construir infraestrutura resiliente, promover a industrialização inclusiva e sustentável e promover a inovação) e 10 (reduzir a desigualdade dentro e entre países) podem ser atingidos se corretamente administrada essa função da IA. Objetivos análogos também podem ser atingidos quando voltamos a aplicação dos entes inteligente artificialmente na saúde e no saneamento.

\subsection{Inteligência Artificial e Saúde: ODS's 3 e 11}

Desde a revolução comunista de 1949 até a virada do milênio, as metrópoles chinesas estavam repletas de bicicletas. Contudo, com as reformas sociais e econômicas no início dos anos 2000 fizeram com que o setor automobilístico ganhasse força, andar de bicicleta tornou-se um estigma para pessoas muito pobres e incapazes financeiramente de utilizar o transporte de quatro rodas.

Esse cenário mudou drasticamente no final de 2015, onde startups de compartilhamento de bicicletas lideradas pela Mobike e Ofo começaram a fornecer dezenas de milhões desses veículos conectadas a internet para distribuí-las pelas principais avenidas da China. A Mobike utilizou a inteligência artificial para criar e equipar suas bicicletas com códigos QR e fechaduras inteligentes conectadas à internet ao redor da roda traseira. Quando o usuário usava o aplicativo da empresa para escanear o código em questão, a trava na roda se abria automaticamente. Assim, os usuários podem andar de um lado par ao outro e deixar a bicicleta onde quiserem para o próximo vir a utilizar. Os custos de um passeio são variáveis de acordo com o percurso em análise e o tempo, mas os subsídios pesados 
SANTOS DIVINO, Sthéfano Bruno. Reflexões sobre a inteligência artificial na agenda 2030 para o desenvolvimento sustentável. Revista Eletrônica Direito e Política, Programa de Pós-Graduação Stricto Sensu em Ciência Jurídica da UNIVALI, Itajaí, v.16, n.1, $1^{0}$ quadrimestre de 2021. Disponível em: www.univali.br/direitoepolitica - ISSN 1980-7791.

significam que, em geral, custam quinze centavos ou menos. Apenas no outono de 2017 a Mobike registrou 22 milhoes de viagens por dia, quase todas realizadas na China. Para ter uma comparação significativa, a Uber naquele mesmo ano realizou cerca de quatro vezes menos o número de viagens globais se comparadas com a Mobike. ${ }^{21}$

Estima-se que 2017 e 2020, a Zona de Desenvolvimento Econômico e Tecnológico de Nanjing invista pelo menos 450 milhões de dólares em desenvolvimento de IA. Dentro desse processo tecnológico, o governo estipula a campanha "inovação e massa e empreendedorismo em massa", que começou em 2015. Desde então, entre 2007 e 2017, a China passou de ter zero linhas ferroviárias de alta velocidade a acumular mais quilômetros que o resto do mundo combinado. ${ }^{22} \mathrm{Com}$ a força de capacitação das empresas de IA envolvidas no segmento, torna-se possível diminuir o estresse diário do intenso tráfego das grandes metrópoles e, da mesma forma, a redução de gases poluentes.

No mesmo sentido, a startup RXThinking está tentando criar e treinando algoritmos médicos de IA para se tornarem super-diagnosticadores. Em vez de substituir os médicos por algoritmos, o aplicativo de diagnóstico de IA do RXThinking os capacita. Ele funciona como um "aplicativo de navegação" para o processo de diagnóstico, aproveitando todo o conhecimento disponível para recomendar o melhor caminho, mas ainda assim permitindo que os médicos conduzam o carro. ${ }^{23}$ Isso aumentaria a certeza do diagnóstico bem como os futuros tratamentos das pessoas enfermas.

Essas tecnologias e aprimoramentos são exemplos de como a IA pode ser utilizada para garantir uma vida saudável e promover o bem-estar para

\footnotetext{
${ }^{21}$ LEE, Kai-Fu. Inteligência Artificial. Rio de Janeiro: Globo Livros, 2019, p. 98.

22 Idem.

23 LEE, Kai-Fu. 'The Algorithm Will See You Now'. AISuperpowers. 2019. https://aisuperpowers.com/blog/kai-fu-lee
} 
SANTOS DIVINO, Sthéfano Bruno. Reflexões sobre a inteligência artificial na agenda 2030 para o desenvolvimento sustentável. Revista Eletrônica Direito e Política, Programa de Pós-Graduação Stricto Sensu em Ciência Jurídica da UNIVALI, Itajaí, v.16, n.1, $1^{0}$ quadrimestre de 2021. Disponível em: www.univali.br/direitoepolitica - ISSN 1980-7791.

todos em todas as idades (ODS 3) e tornar cidades e assentamentos humanos inclusivos, seguros, resilientes e sustentáveis (ODS 11). E não apenas, conexões aparentemente sustentáveis demonstram o âmbito de abrangência ligado a IA capaz de satisfazer outros objetivos da Agenda 2030.

\subsection{Outros impactos da Inteligência Artificial nos ODS's da Agenda 2030}

Um dos primeiros elementos a ser evidenciado é a participação da IA na educação. Como essa em seu desenvolvimento está intrinsecamente ligada ao desenvolvimento tecnológico, principalmente aos aparatos da Tecnologia da Informação e Comunicação (TIC's), é essencial e necessário manter o acesso a esse sistema de recursos de forma equânime.

Em 19 de março de 2018, o governo inaugurou o Centro Nacional de Educação Colaborativa em Robótica (Centre national collaboratif de l'éducation en robotique, RobotsMali) no novo Cité universitaire de Kabala, ao sul de Bamako. Além de ensinar robótica e codificação, o RobotsMali oferece treinamento em IA, eletrônica e Internet das Coisas, bem como em empreendedorismo, marketing, design e gerenciamento de projetos. Como parte de seu Programa de Capacitação para Jovens na STI, o escritório da UNESCO em Bamako forneceu ao RobotsMali material e equipamentos educacionais em 2018, em coordenação com o Departamento de África da UNESCO e com o apoio financeiro da China. Esse material incluiu 40 laptops e kits de robôs e drones para diferentes faixas etárias: crianças até 13 anos, adolescentes e estudantes universitários. O projeto foi implementado em parceria com o Ministério da Educação e o Ministério da Inovação e Pesquisa Científica, juntamente com a Associação Maliana de Engenheiras. O Programa de Capacitação para Jovens da UNESCO em STI também planeja treinar professores nas escolas primárias e secundárias em todo o Mali em 
SANTOS DIVINO, Sthéfano Bruno. Reflexões sobre a inteligência artificial na agenda 2030 para o desenvolvimento sustentável. Revista Eletrônica Direito e Política, Programa de Pós-Graduação Stricto Sensu em Ciência Jurídica da UNIVALI, Itajaí, v.16, n.1, $1^{0}$ quadrimestre de 2021. Disponível em: www.univali.br/direitoepolitica - ISSN 1980-7791.

como ensinar robótica e apoiar escolas que desejam criar seus próprios clubes de robótica. ${ }^{24}$

Outro exemplo de desenvolvimento de IA voltada para educação é o aplicativo VIPKid Teach. Elaborado para conectar professores norteamericanos a estudantes chineses para aulas de inglês on-line, o aplicativo tende a facilitar e reduzir fronteiras através da contínua coleta de dados sobre o envolvimento dos alunos através de análises de expressão fácil e do aparente sentimento. Tais dados são utilizados sem acessar o perfil dos estudantes em si, permitindo as plataformas a filtrar os tipos de professores que são mais compatíveis com o aluno em questão. ${ }^{25}$

Complementando, de forma geral, Vinuesa, R., Azizpour, H., Leite, I. et al. elaboraram um estudo sistemático demonstrando as possíveis incursões da IA no cumprimento dos ODS's bem como seus eventuais reflexos negativos. Sua exibição detalhada pode ser verificada a seguir.

24 UNESCO. Artificial intelligence for sustainable development: challenges and opportunities for UNESCO's science and engineering programmes. 2019, p. 45.

25 VIPKID. https://m.vipkid.com/ 
SANTOS DIVINO, Sthéfano Bruno. Reflexões sobre a inteligência artificial na agenda 2030 para o desenvolvimento sustentável. Revista Eletrônica Direito e Política, Programa de Pós-Graduação Stricto Sensu em Ciência Jurídica da UNIVALI, Itajaí, v.16, n.1, $1^{0}$ quadrimestre de 2021. Disponível em: www.univali.br/direitoepolitica - ISSN 1980-7791.

Figura 2: Resumo dos impactos positivo e negativo da IA nos vários ODS 26
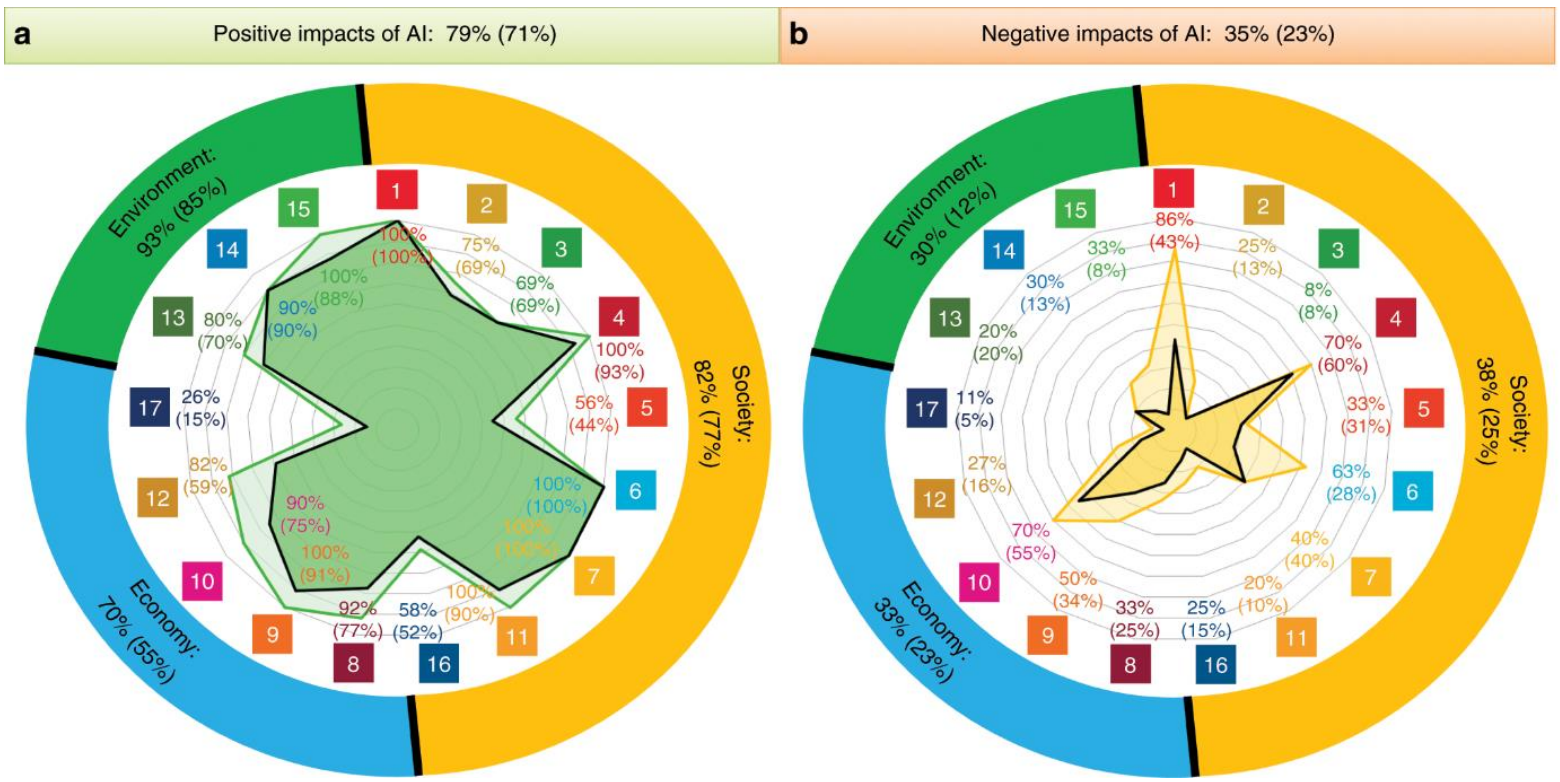

Apesar de descritivamente didático, algumas observações são tecidas. O gráfico do lado esquerdo apresenta os impactos positivos da IA nos 17 ODS's espalhados conforme seus principais aspectos influenciadores (sociedade, meio ambiente e economia). De todos os ODS's os autores preveem uma participação de cerca de $79 \%$ da IA em todos eles. Os principais são os ODS's: 1 (Acabar com a pobreza de todas as formas em todos os lugares); 4 (Garantir uma educação de qualidade inclusiva e equitativa e promover oportunidades de aprendizagem ao longo da vida para todos); 6 (Garantir a disponibilidade e gestão sustentável da água e saneamento para todos); 7 (Garantir acesso a energia acessível, confiável, sustentável e moderna para todos) ${ }^{27}$; 9 (Construir infraestrutura resiliente, promover a industrialização inclusiva e sustentável e promover a inovação)

26 VINUESA, R., AZIZPOUR, H., LEITE, I. et al. The role of artificial intelligence in achieving the Sustainable Development Goals. Nat Commun, v.11, 2020, p. 233. https://doi.org/10.1038/s41467-019-14108-y

27 Nerini, Slob, Engstrom e Trutnevyte demonstram como um modelo de cidade inteligente utilizando uma IA pode reduzir as emissões de gás carbônico dentro das metrópoles, as quais são responsáveis pela emissão de $60 \%$ a $80 \%$ do CO2 presente na atmosfera. FUSO NERINI, F.; SLOB, A.; ERICSDOTTER ENGSTRÖM, R.; TRUTNEVYTE, E. A Research and Innovation Agenda for ZeroEmission European Cities. Sustainability, 2019, 11, 1692. https://doi.org/10.3390/su11061692 
SANTOS DIVINO, Sthéfano Bruno. Reflexões sobre a inteligência artificial na agenda 2030 para o desenvolvimento sustentável. Revista Eletrônica Direito e Política, Programa de Pós-Graduação Stricto Sensu em Ciência Jurídica da UNIVALI, Itajaí, v.16, n.1, $1^{0}$ quadrimestre de 2021. Disponível em: www.univali.br/direitoepolitica - ISSN 1980-7791.

e 14 (Conservar e usar de forma sustentável os oceanos, mares e recursos marinhos para o desenvolvimento sustentável).

Ainda com relação aos aspectos positivos da incrementação da $I A$, os autores elaboram duas figuras que demonstram a participação da IA em cada um dos 17 ODS's, bem como o que essa tecnologia pode fazer para atingir os alvos individualizados expressos em cada um deles. Para aprimorar a compreensão, os dados foram fracionados levando em consideração a área de atuação da IA: se na sociedade ou se na economia.

Figura 3: Avaliação detalhada do impacto da IA nos ODS dentro do grupo Sociedade 28

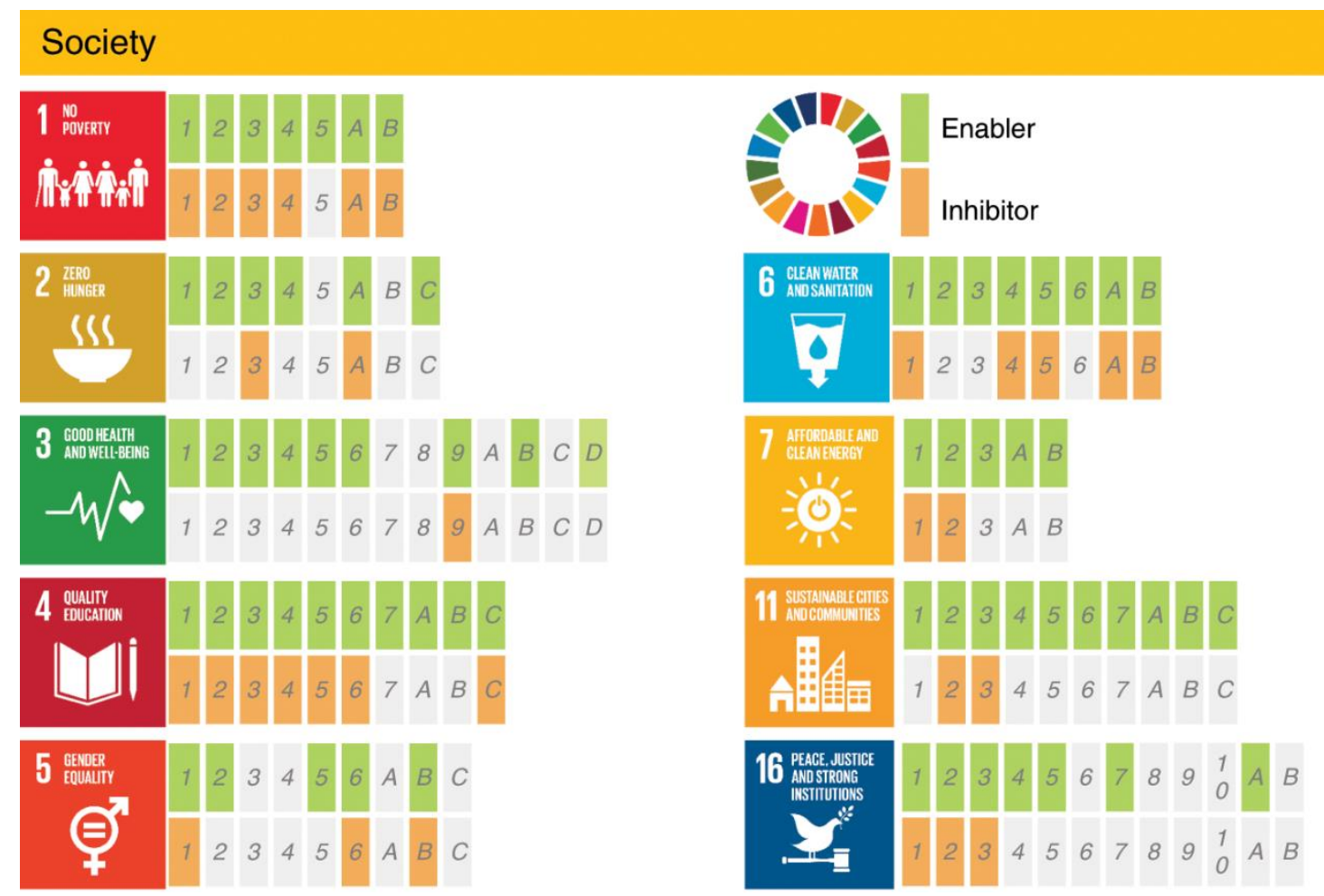

28 VINUESA, R., AZIZPOUR, H., LEITE, I. et al. The role of artificial intelligence in achieving the Sustainable Development Goals. Nat Commun, v.11, 2020, p. 233. https://doi.org/10.1038/s41467-019-14108-y 
SANTOS DIVINO, Sthéfano Bruno. Reflexões sobre a inteligência artificial na agenda 2030 para o desenvolvimento sustentável. Revista Eletrônica Direito e Política, Programa de Pós-Graduação Stricto Sensu em Ciência Jurídica da UNIVALI, Itajaí, v.16, n.1, $1^{0}$ quadrimestre de 2021. Disponível em: www.univali.br/direitoepolitica - ISSN 1980-7791.

Figura 4: Avaliação detalhada do impacto da IA nos ODS dentro do grupo Economia 29

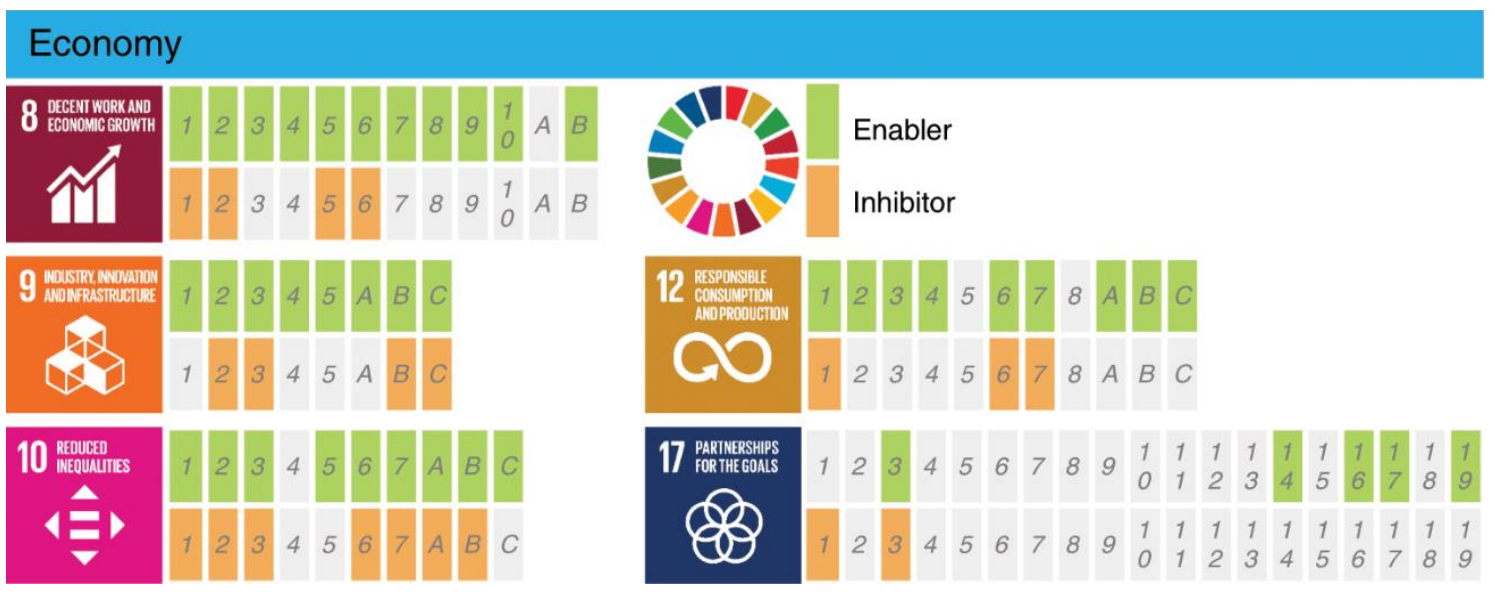

Os dados nos blocos maiores demonstram quais ODS's são pautadas, enquanto os blocos menores expressos em verde, onde a IA participa de forma positiva, ou alaranjado, onde a IA participa de forma negativa, são os targets ou alvos individuais contidos em cada um dos ODS.

A princípio parece evidente o quão positivo a adoção da IA na Agenda 2030 pode ser. Esses aspectos devem ser relevados de maneira a incentivar os países a produção de matéria intelectual voltada para essa área. O que também merece destaque são os impactos negativos capazes de afetar principalmente o setor econômico e parte do setor social. Por exemplo: caso as tecnologias que envolvam IA e o Big Data forem usadas em regiões onde falta escrutínio ético, transparência e controle democrático, elas podem agravar a situação provocando ódio por minorias e influenciar os resultados de eleições. ${ }^{30}$

\footnotetext{
${ }^{29}$ VINUESA, R., AZIZPOUR, H., LEITE, I. et al. The role of artificial intelligence in achieving the Sustainable Development Goals. Nat Commun, v.11, 2020, p. 233. https://doi.org/10.1038/s41467-019-14108-y

30 HELBING, D. \& POURNARAS, E. Society: build digital democracy. Nature, v. 527, 2015, p. 3335).

https://www.nature.com/news/polopoly fs/1.18690!/menu/main/topColumns/topLeftColumn/pdf/5 27033a.pdf
} 
SANTOS DIVINO, Sthéfano Bruno. Reflexões sobre a inteligência artificial na agenda 2030 para o desenvolvimento sustentável. Revista Eletrônica Direito e Política, Programa de Pós-Graduação Stricto Sensu em Ciência Jurídica da UNIVALI, Itajaí, v.16, n.1, $1^{0}$ quadrimestre de 2021. Disponível em: www.univali.br/direitoepolitica - ISSN 1980-7791.

O termo big nudging, por exemplo, surge para representar o uso de Big Data e IA para explorar fraquezas psicológicas para orientar decisões capazes de criar problemas ligados à negativação da coesão social, princípios democráticos e até direitos humanos. ${ }^{31}$ Já existem demonstrações da utilização de IA no cenário consumerista que utiliza o denominado score para previsibilidade e controle de comportamentos sociais. $^{32}$

This type of score is a clear example of threat to human rights due to AI misuse and one of its biggest problems is the lack of information received by the citizens on the type of analyzed data and the consequences this may have on their lives. It is also important to note that AI technology is unevenly distributed: for instance, complex AI-enhanced agricultural equipment may not be accessible to small farmers and thus produce an increased gap with respect to larger producers in more developed economies, consequently inhibiting the achievement of some targets of SDG 2 on zero hunger. ${ }^{33}$

Outro fator que pode afetar negativamente a utilização da IA no setor social para atingir o ODS 5 referente a igualdade de gênero é a insuficiência de dados e pesquisas capazes de avaliar o impacto potencial de tecnologias como algoritmos inteligentes, reconhecimento de imagens ou aprendizado reforçado sobre a discriminação contra mulheres e minorias. ${ }^{34}$

Com relação ao aspecto econômico, apesar da existência de aspectos positivos inicialmente já demonstrados, a literatura aponta para um eventual crescimento da desigualdade, impactando significativamente os ODS 8 (trabalho decente e crescimento econômico), 9 (indústria, inovação

\footnotetext{
31 HELBING D. ET AL. (2019) Will Democracy Survive Big Data and Artificial Intelligence?. In: HELBING D. (Ed) Towards Digital Enlightenment. Springer, Cham. https://doi.org/10.1007/9783-319-90869-4 7

32 NAGLER, J., VAN DEN HOVEN, J. \& HELBING, D. in Towards Digital Enlightenment. Springer International Publishing, 2019, p. 41-46. https://doi.org/10.1007/978-3-319-90869-4_5

33 VINUESA, R., AZIZPOUR, H., LEITE, I. et al. The role of artificial intelligence in achieving the Sustainable Development Goals. Nat Commun, v.11, 2020, p. 233. https://doi.org/10.1038/s41467-019-14108-y

34 VINUESA, R., AZIZPOUR, H., LEITE, I. et al. The role of artificial intelligence in achieving the Sustainable Development Goals. Nat Commun, v.11, 2020, p. 233. https://doi.org/10.1038/s41467-019-14108-y.
} 
SANTOS DIVINO, Sthéfano Bruno. Reflexões sobre a inteligência artificial na agenda 2030 para o desenvolvimento sustentável. Revista Eletrônica Direito e Política, Programa de Pós-Graduação Stricto Sensu em Ciência Jurídica da UNIVALI, Itajaí, v.16, n.1, $1^{0}$ quadrimestre de 2021. Disponível em: www.univali.br/direitoepolitica - ISSN 1980-7791.

e infraestrutura) e 10 (redução das desigualdades). ${ }^{35}$ Brynjolfsson e McAfee $^{36}$ postulam a ideia de que a IA pode exacerbar a desigualdade também dentro das nações. Ao substituir empregos antigos por empregos que exigem mais habilidades, a tecnologia recompensa desproporcionalmente os instruídos. Isso pode se dar em virtude da concentração de cientistas da área em determinados países. Segundo relatório recente da Unesco, cerca de 49,1\% dos estudantes de doutorado que estão envoltos em ciências ou engenharia da computação atreladas ao desenvolvimento de IA são estadunidenses.

35 COCKBURN, I., HENDERSON, R. \& STERN, S. The Impact of Artificial Intelligence on Innovation. NBER, 2018. https://doi.org/10.3386/w24449 BRYNJOLFSSON, E. \& MCAFEE, A. The Second Machine Age: Work, Progress, and Prosperity in a Time of Brilliant Technologies. W. W. Norton \& Company, 2014.

36 Idem. 
SANTOS DIVINO, Sthéfano Bruno. Reflexões sobre a inteligência artificial na agenda 2030 para o desenvolvimento sustentável. Revista Eletrônica Direito e Política, Programa de Pós-Graduação Stricto Sensu em Ciência Jurídica da UNIVALI, Itajaí, v.16, n.1, $1^{0}$ quadrimestre de 2021. Disponível em: www.univali.br/direitoepolitica - ISSN 1980-7791.

Figura 6: Distribuição de doutorandos internacionais matriculados em programas de ciência e engenharia por país anfitrião, $2012(\%)^{37}$

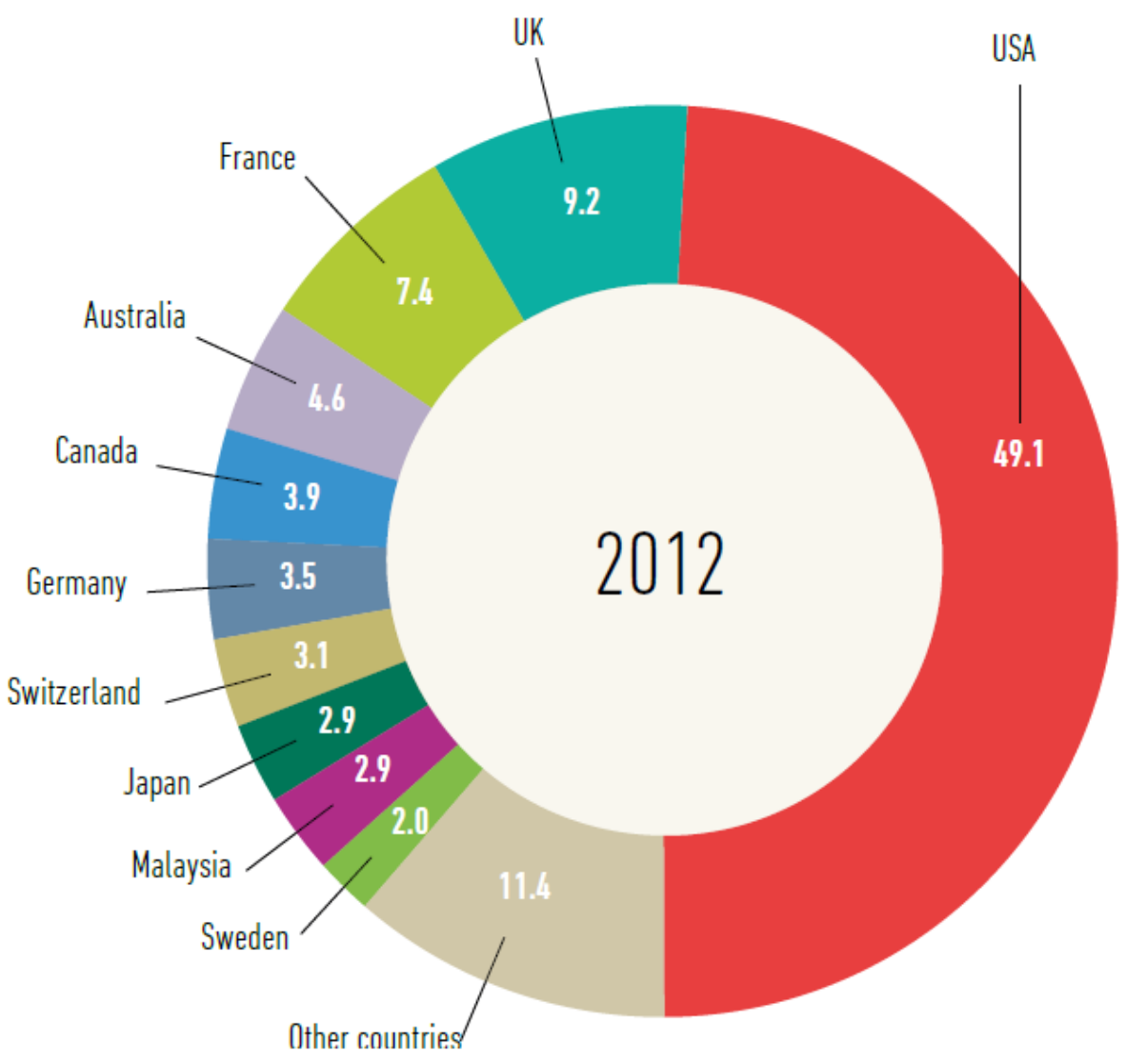

Além disso, Conforme dados da Organização para Cooperação e Desenvolvimento Econômico ${ }^{38}$ (OCDE, 2017), que analisou o comportamento de patenteamento das 2.000 empresas mais importantes em pesquisa e desenvolvimento entre 2012 e 2014, constatou que no cenário contemporâneo existe uma ampla concentração de patentes de IA em empresas multinacionais sediadas em apenas sete economias, o que representava $93 \%$ das patentes de IA registradas nos cinco principais

37 UNESCO. Artificial intelligence for sustainable development: challenges and opportunities for UNESCO's science and engineering programmes. 2019, p. 37.

${ }^{38}$ OECD. Science, Technology and Innovation Scoreboard 2017: the Digital Transformation. Organisation for Economic Co-operation and Development, Paris, 2017. 
SANTOS DIVINO, Sthéfano Bruno. Reflexões sobre a inteligência artificial na agenda 2030 para o desenvolvimento sustentável. Revista Eletrônica Direito e Política, Programa de Pós-Graduação Stricto Sensu em Ciência Jurídica da UNIVALI, Itajaí, v.16, n.1, $1^{0}$ quadrimestre de 2021. Disponível em: www.univali.br/direitoepolitica - ISSN 1980-7791.

escritórios de patentes. ${ }^{39}$ São eles: Japão (33\%), República da Coréia (20\%), EUA (18\%), Província de Taiwan da China (9\%), China (8\%), Alemanha (3\%) e França (2\%). ${ }^{40}$

Figura $7^{41}$

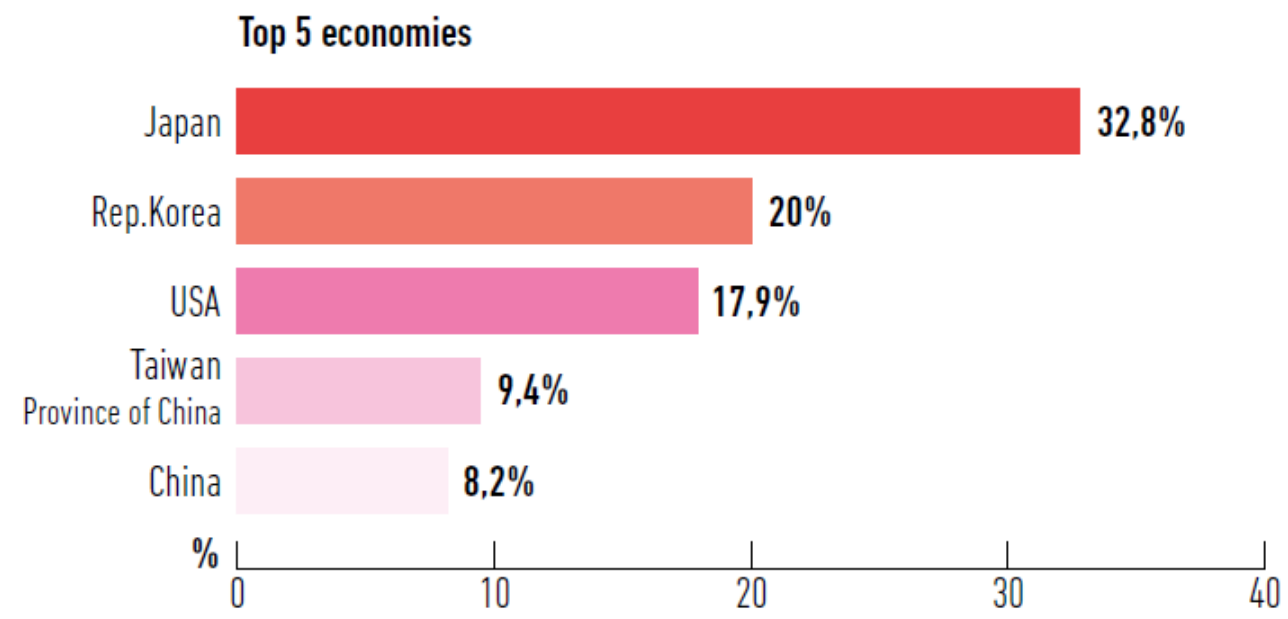

39 Patents registered with the top five patent families: European Patent Office, Japanese Patent Office, Korean Intellectual Property Office, State Intellectual Property Office of the People's Republic of China and the US Patent and Trademark Office.

40 UNESCO. Artificial intelligence for sustainable development: challenges and opportunities for UNESCO's science and engineering programmes. 2019, p. 30.

Para mais, vide em UNESCO. Towards a monopolization of research in artificial intelligence? Natural Sciences $\quad$ Sector. $2018 . \quad$ www.unesco.org/new/en/naturalsciences/sciencetechnology/single-view-scpolicy/news/towards_a_monopolization_of_research_in_artificial_intellige 41 Idem. 
SANTOS DIVINO, Sthéfano Bruno. Reflexões sobre a inteligência artificial na agenda 2030 para o desenvolvimento sustentável. Revista Eletrônica Direito e Política, Programa de Pós-Graduação Stricto Sensu em Ciência Jurídica da UNIVALI, Itajaí, v.16, n.1, 10 quadrimestre de 2021. Disponível em: www.univali.br/direitoepolitica - ISSN 1980-7791.

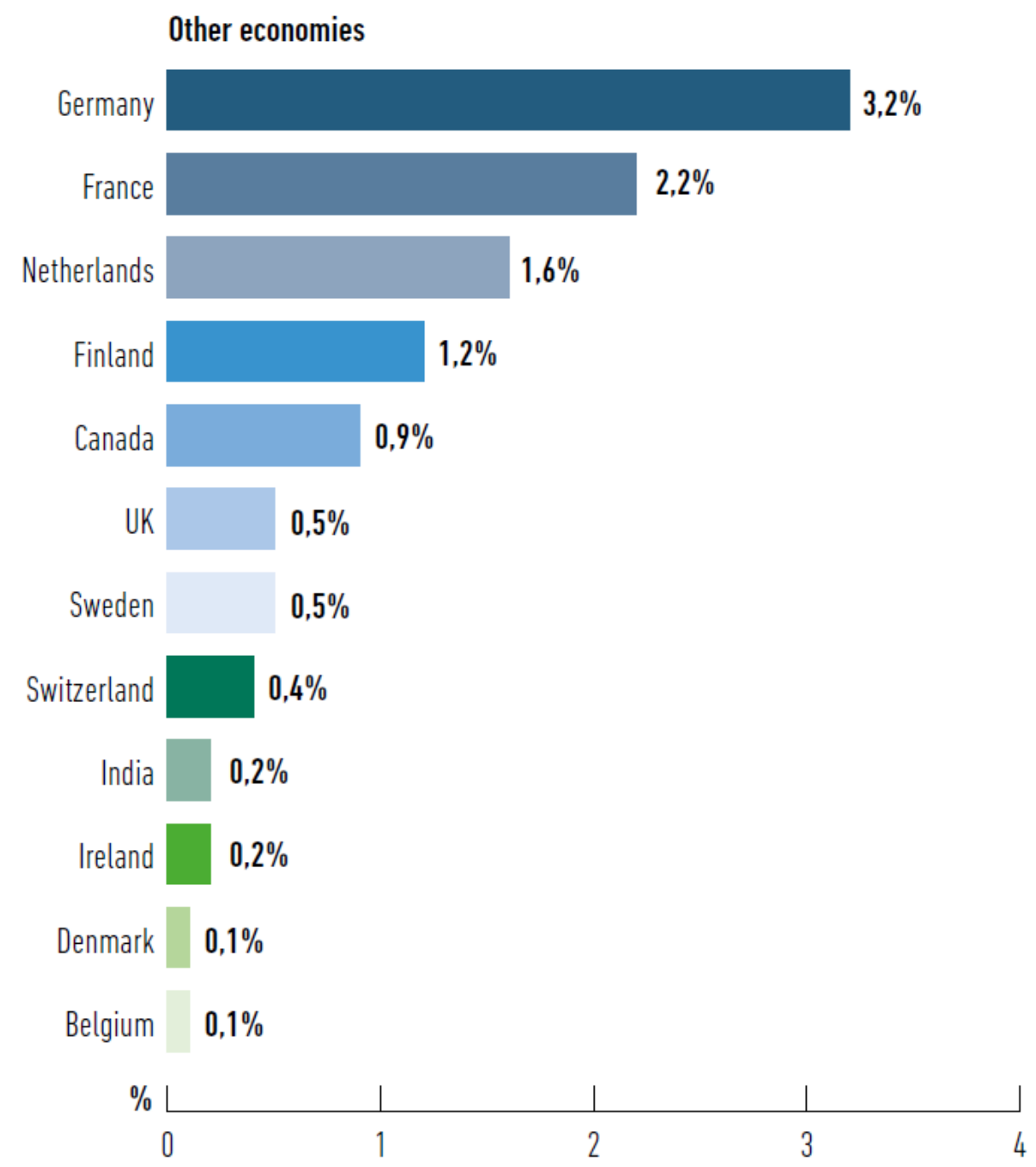

Outro aspecto negativo que a IA pode trazer está no uso da mídia social, selecionando e fracionando conteúdo especificamente adequado aos ideais de seus usuários que podem ser analisadas pelo programa e detectadas como pré-concebidas. Tal conduta pode levar à polarização política e afetar a coesão social, com consequências no contexto do ODS 10 sobre a redução das desigualdades. ${ }^{42}$

É necessário ter em conta que esses dados são passíveis de se tornarem flexíveis. A disciplina de fato determina um circuito novo em relação até que ponto o momento da inteligência artificial pode garantir positividade e

42 FRANCESCATO, D. Globalization, artificial intelligence, social networks and political polarization: new challenges for community psychologists. Commun. Psychol. Glob. Perspect. 4, 2018, p. 2041. http://siba-ese.unisalento.it/index.php/cpgp/article/viewFile/17684/15882 
elasticidade das condutas sociais. de um certo ponto de vista, poder-se-ia concluir que tais predições mencionadas constituem um legado quase inviável de uma realidade de continuo movimento e desenvolvimento. Sem querer colocar em discussão a validade dessa argumentação, tornam-se necessário refletir sobre o papel que essas alegações podem ter se consideradas válidas e concretizadas em um futuro próximo. Os códigos de deontologia e de boa conduta, ligados aos aparatos éticos, podem fornecer determinados perfis normativos ligados a posições mercadológicas e políticas. Do ponto de vista do processo de produção da justiça, estes códigos às vezes são promovidos pelos próprios responsáveis pelo desenvolvimento das tecnologias. É neste momento que o auxílio de uma perspectiva jurídica torna-se necessário. O Direito tende a atuar como garantidor das políticas e adereços positivos elencados pela IA e, ao mesmo tempo, diminuir ou eliminar as predições negativas. Por essa razão, o próximo tópico tende a abordar a correlação entre as então existentes legislações sobre inteligência artificial e demonstrar a longa fronteira na qual os sistemas políticos e jurídicos encontram-se frente a vinheta produzida pelas novas tecnologias.

\section{INTELIGÊNCIA ARTIfICIAL NA PROGRAMAÇÃO E PRODUÇÃO NORMATIVA: O PAPEL DO DIREITO PARA O CUMPRIMENTO DA AGENDA 2030}

A representação de políticas normativas voltadas para o desenvolvimento da IA ganha destaque em alguns países. Em março de 2017 o governo federal do Canadá foi o primeiro país do mundo a anunciar um investimento 
SANTOS DIVINO, Sthéfano Bruno. Reflexões sobre a inteligência artificial na agenda 2030 para o desenvolvimento sustentável. Revista Eletrônica Direito e Política, Programa de Pós-Graduação Stricto Sensu em Ciência Jurídica da UNIVALI, Itajaí, v.16, n.1, $1^{0}$ quadrimestre de 2021. Disponível em: www.univali.br/direitoepolitica - ISSN 1980-7791.

de US \$ 125 milhões destinado à uma estratégia nacional de inteligência artificial (IA) a ser concretizada nos próximos cinco anos. ${ }^{43}$

A Estratégia Pan-Canadense de Inteligência Artificial baseia-se em uma parceria entre o Instituto Canadense de Pesquisa Avançada (CIFAR Canadian Institute for Advanced Research) e os três centros de excelência: o Alberta Machine Intelligence Institute (AMII) em Edmonton, o Vector Institute em Toronto e o Montreal Institute for Learning Algorithms (Mila) em Montreal. Juntos, eles fornecem suporte, recursos e talento para inovação e investimento em IA.

Embora sejam conhecidos os aspectos positivos da aplicação da IA, o governo do Canadá também está preocupado com os reflexos da atividade computacional nos empregos, privacidade, segurança digital, democracia e ética. Por esse motivo, o país está assumindo um papel de liderança nas conversas internacionais, buscando entender as implicações sociais da IA. Em março de 2018, o Governo de Quebec propôs a criação de uma Organização Mundial da Inteligência Artificial (Omia), uma organização intergovernamental dedicada a promover o consenso entre os Estados membros sobre os padrões e práticas que devem governar as aplicações da IA. ${ }^{44}$

Ainda em março de 2018, o Fonds de recherche du Québec, em conjunto com o Ministério da Economia, Ciência e Inovação, lançou um convite à apresentação de propostas a todas as universidades de Quebec para a criação de um observatório internacional sobre o impacto social da IA e das tecnologias digitais ${ }^{45}$. Esse observatório será utilizado para apoiar pesquisas

43 UNESCO. Canada first to adopt strategy for artificial intelligence. 2018. http://www.unesco.org/new/en/member-states/singleview/news/canada first to adopt strategy for artificial intelligence/

${ }^{44}$ Idem.

45 SÉVIGNY, B. Québec lays the groundwork for a world observatory on the social impacts of artificial intelligence and digital Technologies. 2018. http://www.scientifique-enchef.gouv.qc.ca/en/nouvelles/quebec-jette-bases-dun-observatoire-mondial-impacts-societaux-delintelligence-artificielle-numerique/ 
SANTOS DIVINO, Sthéfano Bruno. Reflexões sobre a inteligência artificial na agenda 2030 para o desenvolvimento sustentável. Revista Eletrônica Direito e Política, Programa de Pós-Graduação Stricto Sensu em Ciência Jurídica da UNIVALI, Itajaí, v.16, n.1, 10 quadrimestre de 2021. Disponível em: www.univali.br/direitoepolitica - ISSN 1980-7791.

prospectivas, mobilização de conhecimento e engajamento público em torno das implicações sociais da IA. A criação do observatório se baseia em um movimento de base que se formou ao longo do ano de 2017, com a organização do Fórum sobre Desenvolvimento Socialmente Responsável da IA, que adotou a Declaração de Montreal para o Desenvolvimento Responsável da Inteligência Artificial ${ }^{46} .47$

Em junho de 2018, antes das reuniões do G7 em Charlevoix, Quebec, o primeiro-ministro Justin Trudeau e o presidente Emmanuel Macron emitiram uma declaração conjunta Canadá-França sobre a IA.

Canada and France affirm that artificial intelligence is a revolution whose impact is being felt more and more each day. In the near future, it will influence all human activity, providing unprecedented economic and social benefits. Innovations in artificial intelligence technologies will create new sources of economic growth that could make our economies more competitive, inclusive and sustainable, create jobs and shape a better future for all our citizens. ${ }^{48}$

Eles se comprometeram a estabelecer um grupo de estudo internacional que reunisse especialistas de vários países e setores e forneça um fórum para compartilhar análises e melhores práticas e fornecer capacidade de previsão e coordenação. Uma observação importante que pode ser realizada é que os programas governamentais em questão tentem a fazer

\footnotetext{
${ }^{46}$ MONTREAL. Montréal Declaration for a Responsible Development of Artificial Intelligence. 2020. https://www.montrealdeclaration-responsibleai.com/

"A Declaração de Montreal, delimita, segundo a literatura especializada, o primeiro instrumento dedicado a explorar princípios e regras centradas na tríade governança, ética e responsabilidades da IA, opondo-se à concepção predominante em torno das aplicações tendenciosas, aparentemente neutras e opacas das tecnologias empregadas na robótica e aprendizado de máquina". POLIDO, Fabrício Bertini Pasquot. Inteligência artificial entre estratégias nacionais e a corrida regulatória global: rotas analíticas para uma releitura internacionalista e comparada. Rev. Fac. Direito UFMG, $\begin{array}{lllll}\text { Belo Horizonte, } & \text { n. 76, pp. 229-256, jan./jun. } 2020 .\end{array}$ https://www.direito.ufmg.br/revista/index.php/revista/article/view/2067/1931

STARK Luke, PYLYSHYN, Zenon W. Artificial Intelligence (AI), cit.; 'Here's how Canada can be a global leader in ethical $\mathrm{AI}^{\prime}$. In: The Conversation. 2018. Disponível em $<$ https://theconversation.com/heres-how-canada-can-be-a-global-leader-in-ethical-ai-90991>.

47 POLIDO, Fabrício Bertini Pasquot. Inteligência artificial entre estratégias nacionais e a corrida regulatória global: rotas analíticas para uma releitura internacionalista e comparada. Rev. Fac. Direito UFMG, Belo Horizonte, n. 76, pp. 229-256, jan./jun. 2020. https://www.direito.ufmg.br/revista/index.php/revista/article/view/2067/1931

48 BAINS, N. S.; VIDAL, F. Canada-France Statement on Artificial Intelligence. 2018. https://www.international.gc.ca/world-monde/international relationsrelations internationales/europe/2018-06-07-france_ai-ia_france.aspx?lang=eng
} 
SANTOS DIVINO, Sthéfano Bruno. Reflexões sobre a inteligência artificial na agenda 2030 para o desenvolvimento sustentável. Revista Eletrônica Direito e Política, Programa de Pós-Graduação Stricto Sensu em Ciência Jurídica da UNIVALI, Itajaí, v.16, n.1, $1^{0}$ quadrimestre de 2021. Disponível em: www.univali.br/direitoepolitica - ISSN 1980-7791.

uma eliminação de uma abordagem puramente mercadológica dos traços que são deixados nas transações comerciais que envolvem IA. Tal tecnologia, conforme as diretrizes canadenses, tendem a ser balanceadas e ponderadas para atendimento de interesses humanamente relevantes. A abordagem produzida, portanto, deve ser reconduzida ao âmbito e ao aspecto humanitário. Fato esse que demonstra uma preocupação pelo governo der que o sujeito esteja assumindo uma posição de importância e relevância no desenvolvimento dessas novas tecnologias.

No mesmo escopo, o Reino Unido apresenta uma política com investimentos significativos no desenvolvimento da IA. O Artificial Intelligence Sector Deal é um pacote de $£ 1$ bilhão de apoio do governo e da indústria que objetiva aumentar e consagrar a posição global do Reino Unido como líder no desenvolvimento de IA e tecnologias relacionadas. O projeto está adotando ações tangíveis para promover estratégias voltadas para indústria da IA e dos desafios dos dados para garantir que o Reino Unido seja o principal destino para inovação e investimento em IA. ${ }^{49}$

Objetivando criar uma ambientação segura e atrair investidores para a área, o projeto em questão conta com a adoção de um conselho formado com Tabitha Goldstaub, como presidente do Conselho da AI, e Dame Wendy Hall, como campeã de habilidades da AI, para fortalecer a conversa entre a academia, a indústria e o setor público. Além disso, existe um escritório próprio para assumir a responsabilidade de supervisionar o projeto e cumprir os compromissos estabelecidos inicialmente estabelecidos. Para auxiliar esse desenvolvimento, estabeleceu-se o Centro de Ética e Inovação de Dados para fornecer consultoria independente e especializada sobre as

49 UNITED KINGDOM. AI Sector Deal One Year On. 2019. https://assets.publishing.service.gov.uk/government/uploads/system/uploads/attachment data/fil e/819331/AI Sector Deal One Year_On Web.pdf 
SANTOS DIVINO, Sthéfano Bruno. Reflexões sobre a inteligência artificial na agenda 2030 para o desenvolvimento sustentável. Revista Eletrônica Direito e Política, Programa de Pós-Graduação Stricto Sensu em Ciência Jurídica da UNIVALI, Itajaí, v.16, n.1, 10 quadrimestre de 2021. Disponível em: www.univali.br/direitoepolitica - ISSN 1980-7791.

medidas necessárias para permitir e garantir usos seguros, éticos e inovadores da IA e das tecnologias orientadas a dados. 50

A preocupação com o desenvolvimento humanista e a utilização da IA no escopo social chamou a atenção da House of Lords para elaboração de um documento contendo diretrizes, ou ao menos indicações, para o governo mitigar os riscos dessa tecnologia quando inserta na sociedade. A cartilha leva em considerações os prováveis riscos em torno da responsabilidade civil e responsabilidade criminal que os entes artificialmente inteligentes podem trazer caso sejam inclusos na sociedade sem eventuais regulamentações. Para tanto, deve-se ao menos mitigar ou prever as hipóteses em que a IA esteja em mal funcionamento ou não atenda o escopo inicialmente pretendido, objetivando facilitar as discussões jurídicas futuras em torno dessa possível problemática. ${ }^{51}$

\footnotetext{
50 Idem.

51 Mitigating the risks of artificial intelligence Legal liability Recommendations 55-56 IV. In our opinion, it is possible to foresee a scenario where AI systems may malfunction, underperform or otherwise make erroneous decisions which cause harm. In particular, this might happen when an algorithm learns and evolves of its own accord. It was not clear to us, nor to our witnesses, whether new mechanisms for legal liability and redress in such situations are required, or whether existing mechanisms are sufficient. (Paragraph 317) Ivi. Clarity is required. We recommend that the Law Commission consider the adequacy of existing legislation to address the legal liability issues of AI and, where appropriate, recommend to Government appropriate remedies to ensure that the law is clear in this area. At the very least, this work should establish clear principles for accountability and intelligibility. This work should be completed as soon as possible. (Paragraph 318) 95. Government welcomes the above recommendation and the acknowledgement of potential errors produced through artificial intelligence technologies and their potential implications. We believe that artificial intelligence technologies should serve people, businesses, and sectors beneficially and, where any outcomes resulting from errors are detrimental to these groups, remedial action should be undertaken. The Office for Artificial Intelligence, Centre for Data Ethics and Innovation, and the AI Council will take these concerns into consideration and, as appropriate, engage the Law Commission on best course of action. Criminal misuse of artificial intelligence and data Recommendations 57 - 59 Ivii. The potential for well-meaning AI research to be used by others to cause harm is significant. AI researchers and developers must be alive to the potential ethical implications of their work. The Centre for Data Ethics and Innovation and the Alan Turing Institute are well placed to advise researchers on the potential implications of their work, and the steps they can take to ensure that such work is not misused. However, we believe additional measures are required. (Paragraph 328) 96. A response to recommendation 57 can be found in the final section of this document. 32 Iviii. We recommend that universities and research councils providing grants and funding to AI researchers must insist that applications for such money demonstrate an awareness of the implications of the research and how it might be misused, and include details of the steps that will be taken to prevent such misuse, before any funding is provided. (Paragraph 329). UNITED KINGDOM. Government response to House of Lords Artificial Intelligence Select Committee's Report on AI in the UK: Ready, Willing and Able?. 2018. https://www.parliament.uk/documents/lordscommittees/Artificial-Intelligence/AI-Government-Response2.pdf
} 
SANTOS DIVINO, Sthéfano Bruno. Reflexões sobre a inteligência artificial na agenda 2030 para o desenvolvimento sustentável. Revista Eletrônica Direito e Política, Programa de Pós-Graduação Stricto Sensu em Ciência Jurídica da UNIVALI, Itajaí, v.16, n.1, $1^{0}$ quadrimestre de 2021. Disponível em: www.univali.br/direitoepolitica - ISSN 1980-7791.

A China também apresenta um plano de desenvolvimento voltado para a corrida da conquista da liderança da IA. O denominado AI development plan (AIDP) pretende colocar a China como percursora global nesse ramo até 2030. Publicado em 2017, o Plano de Desenvolvimento para uma Nova Geração de Inteligência Artificial do Conselho de Estado chinês compartilhou algumas das recomendações do plano expresso pela Casa Branca em 2016, até então presidida por Barack Obama. Pretende-se aumentar o financiamento para pesquisa, intensificar a cooperação civil-militar e fazer investimentos para diminuição das eventuais perturbações sociais. ${ }^{52}$

De forma complementar, as questões legais e de governança estão intrinsecamente ligadas ao desenvolvimento da IA chinesa. Juristas e governantes chineses têm prestado cada vez mais atenção às questões de governança da IA, desde questões de curto prazo até riscos existenciais. Sob uma seção sobre "Medidas de salvaguarda", o plano de IA do Conselho de Estado estabelece uma estrutura para o desenvolvimento de leis, regulamentos e normas éticas para utilização da IA. Contudo, os responsáveis pelo AIDP não se envolvem apenas com contratos de curto prazo problemas de segurança da IA. Existe uma certa preferência e desejo de reformas no sistema legal para lidar com os efeitos da IA na responsabilidade criminal, privacidade, direitos de propriedade intelectual e segurança da informação. As medidas de normatização prospectiva incluem estruturas de vários níveis que determinam a moralidade de vários sistemas de IA, estruturas éticas para colaboração homem-máquina e códigos de conduta para pesquisadores, desenvolvedores e designers de produtos de IA. O principal objetivo do plano é a regulamentação em território nacional, mas reconhece que a adoção de eventuais tratados internacionais é crucial para o desenvolvimento da IA da China. Para esse fim, também pede à China que "fortaleça a pesquisa sobre problemas comuns globais" - em

52 LEE, Kai-Fu. Inteligência Artificial. Rio de Janeiro: Globo Livros, 2019, p. 121. 
SANTOS DIVINO, Sthéfano Bruno. Reflexões sobre a inteligência artificial na agenda 2030 para o desenvolvimento sustentável. Revista Eletrônica Direito e Política, Programa de Pós-Graduação Stricto Sensu em Ciência Jurídica da UNIVALI, Itajaí, v.16, n.1, $1^{0}$ quadrimestre de 2021. Disponível em: www.univali.br/direitoepolitica - ISSN 1980-7791.

particular, menciona problemas de funcionamento de robôs, nos quais os robôs divergem das metas predefinidas de seus fabricantes como um problema comum - e "aprofundem a cooperação internacional em leis e regulamentos de inteligência artificial, regras internacionais etc., para lidar em conjunto com os desafios globais. ${ }^{53}$

Esses são alguns dos categóricos exemplos que estão sendo criados quando emerge na sociedade uma nova construção humana capaz de alterar significativamente as disposições sociais. o papel e a função do direito devem ser utilizados como função intermediária frequentemente presente nessa indispensável relação. Na corrida para atingir o primeiro lugar do desenvolvimento da IA, os vencedores podem e nós corremos o risco de ter um mercado monopolizado. Dessa forma, faz-se necessário a utilização da disciplina jurídica para manter a conexão entre a imersão das novas tecnologias e as organizações geradas pelo seu resultado. Não se objetiva pautar ou ao menos cercear os rendimentos dos envolvidos. Pelo contrário, pretende-se aplicar a IA como instrumento capaz de atingimento do próprio desenvolvimento sustentável destinado à manutenção de um mundo sustentável.

A novidade é radical. Os riscos da sociedade ligada ao desenvolvimento dos entes inteligentes artificialmente ligam-se tradicionalmente ao uso político e mercadológico de poder. Nessa perspectiva vai se delineando a ideia de controle, que deve ser relativizada pelo conjunto e pela coordenação entre países envolvidos nesse cenário. Se levarmos em consideração os modelos de funcionalidade de uma inteligência artificial, a idealização do compartilhamento dos algoritmos utilizados na sua execução é algo extremamente benéfico aos demais sujeitos, inclusive aos desenvolvedores, já que tal código poderá ser aperfeiçoado pelos sujeitos

53 DING, J. How China Seeks to Govern AI. Medium. 2018. Disponível em https://medium.com/@ChallengesFnd/how-china-seeks-togovern- ai-baf1c0cd1a54 
SANTOS DIVINO, Sthéfano Bruno. Reflexões sobre a inteligência artificial na agenda 2030 para o desenvolvimento sustentável. Revista Eletrônica Direito e Política, Programa de Pós-Graduação Stricto Sensu em Ciência Jurídica da UNIVALI, Itajaí, v.16, n.1, $1^{0}$ quadrimestre de 2021. Disponível em: www.univali.br/direitoepolitica - ISSN 1980-7791.

em rede para uma melhor fluidez correlacionada a capacidade de resposta da IA.

Portanto, a função do Direito neste cenário não é atuar como ferramenta para dificultar a aplicação da IA na sociedade, mas como auxiliar, coordenador e coadjuvante capaz de observar e apenas ser ativo quando necessário. Os dados em pauta assumem uma certa previsão de que a IA tende a crescer nos EUA, no Reino Unido ou na China. E é justamente a caracterização dessa tecnologia como recurso capaz de criar um exame completo das dinâmicas atuais para demonstrar que estamos entrando em uma situação em que se não houver coparticipação e envolvimento dos entes governamentais soberanos, o invólucro será rompido e os efeitos negativos tendem a aumentar. O direito, portanto, deve funcionar para manutenção dos limites de planejamento e justiça.

\section{CONSIDERAÇÕES FINAIS}

Palavras como certo ou errado parecem não ser corretamente empregadas quando abordamos a temática inteligência artificial. disto nasce a inquietante constatação da imensa dificuldade em representar a realidade através da linguagem. Viável ou não viável talvez sejam os termos que trazem maior crédito a esse ramo se considerados os benefícios e malefícios dessas novas tecnologias.

Demonstrou-se no discorrer argumentativo a existência de possíveis atividades voltadas à saúde, educação e economia em que a inteligência artificial pode atuar como percursor da Agenda 2030. A associação entre empresas voltadas para a instauração de cidades inteligentes pode reduzir drasticamente o consumo de água e energia através de um melhor gerenciamento. No mesmo sentido, a introdução desse tipo de tecnologia no âmbito da agricultura pode facilitar o desenvolvimento de pequenas 
SANTOS DIVINO, Sthéfano Bruno. Reflexões sobre a inteligência artificial na agenda 2030 para o desenvolvimento sustentável. Revista Eletrônica Direito e Política, Programa de Pós-Graduação Stricto Sensu em Ciência Jurídica da UNIVALI, Itajaí, v.16, n.1, $1^{0}$ quadrimestre de 2021. Disponível em: www.univali.br/direitoepolitica - ISSN 1980-7791.

comunidades capazes de afetar ODS considerados essenciais para a Agenda 2030. Não é por acaso, também, que no setor de saúde uma inteligência artificial pode auxiliar profissionais a diagnosticar melhor as enfermidades de seus pacientes. Todos esses exemplos e os demais trabalhados evidenciam a potência de enquadramento do mundo globalizado, tanto no espaço físico quanto no ciberespaço, da conexão entre IA e a sociedade. Mas quem dita as regras desse mundo global?

Verificaram-se críticas acerca da monopolização do mercado de inteligência artificial, bem como atitudes éticas e morais envolvendo questionamentos ligados à responsabilidade de suas condutas. Esse cenário enseja uma insistência na necessidade de uma série de ações públicas e coletivas a nível nacional e internacional para reduzir e tornar simbólico essa ameaça constitutiva virtual. Ficou claro como essas diretrizes já estão sendo adotadas através de normativos expressos no Canadá e no Reino Unido. Muitas são as garantias pretendidas pela proteção da sociedade frente a esse risco. Os países envolvidos não objetivam trazer uma maior discrepância econômica e social para sua nação. E esse é um dos motivos pelos quais os investimentos em pauta estão sendo realizados pelos próprios governos. Assim, os resultados obtidos podem ser entregues para a sociedade em questão.

Caso as problemáticas se influências negativas da IA sejam simplesmente ignoradas, existe de fato um alto potencial de que essa tecnologia funcione como retrocesso a Agenda 2030. Por esse motivo compete ao Direito, principalmente atuando através de disposições e políticas públicas satisfazer ou ao menos deixar de forma equitativa o desenvolvimento econômico e social. Em outros termos, não se pode postular a indiferença do quadro tradicional dos direitos ao novo ambiente sustentável, mantendo critérios meramente mercadológicos ativos. Portanto, espera-se uma 
SANTOS DIVINO, Sthéfano Bruno. Reflexões sobre a inteligência artificial na agenda 2030 para o desenvolvimento sustentável. Revista Eletrônica Direito e Política, Programa de Pós-Graduação Stricto Sensu em Ciência Jurídica da UNIVALI, Itajaí, v.16, n.1, $1^{0}$ quadrimestre de 2021. Disponível em: www.univali.br/direitoepolitica - ISSN 1980-7791.

constituição humanitária das atividades envoltas pela IA para o cumprimento da Agenda 2030.

\section{REFERÊNCIAS DAS FONTES CITADAS}

BAINS, N. S.; VIDAL, F. Canada-France Statement on Artificial Intelligence. $2018 . \quad$ https://www.international.gc.ca/worldmonde/international relations-relations internationales/europe/2018-0607-france ai-ia france.aspx?lang=eng

BREWSTER, S. Wilting shrubs? Diagnose plant diseases with an app. MIT Technology Review. 2016. https://www.technologyreview.com/s/602792/wilting-shrubs-diagnoseplant-diseases-with-an-app/

BRYNJOLFSSON, E. \& MCAFEE, A. The Second Machine Age: Work, Progress, and Prosperity in a Time of Brilliant Technologies. W. W. Norton \& Company, 2014.

CHUCK, Gill. Artificial intelligence could help farmers diagnose crop diseases. 2017.

http://news.psu.edu/story/429727/2016/10/04/research/artificialintelligence-couldhelp-farmers-diagnose-crop-diseases

COCKBURN, I., HENDERSON, R. \& STERN, S. The Impact of Artificial Intelligence on Innovation. NBER, 2018. https://doi.org/10.3386/w24449

DING, J. How China Seeks to Govern. AI. Medium. September 5, 2018. Disponível em https://medium.com/@ChallengesFnd/how-china-seekstogovern- ai-baf1c0cd1a54

FRANCESCATO, D. Globalization, artificial intelligence, social networks and political polarization: new challenges for community psychologists. CREFERÊNCIA DAS FONTES CITADAS. 4, 2018, p. 20-41. http://sibaese.unisalento.it/index.php/cpgp/article/viewFile/17684/15882

FUSO NERINI, F.; SLOB, A.; ERICSDOTTER ENGSTRÖM, R.; TRUTNEVYTE, E. A Research and Innovation Agenda for Zero-Emission European Cities. Sustainability 2019, 11, 1692. https://doi.org/10.3390/su11061692

GORALSKI, Margareth A.; TAN, Tay Keong. Artificial intelligence and sustainable development. The International Journal of Management Education. Vol. 18, 2020. 
HELBING D. ET AL. Will Democracy Survive Big Data and Artificial Intelligence? In: HELBING D. (EDS) Towards Digital Enlightenment. Springer, Cham, 2019. https://doi.org/10.1007/978-3-319-90869-4 7

HELBING, D. \& POURNARAS, E. Society: build digital democracy. Nature, 527, 2015 , p. 33-34. https://www.nature.com/news/polopoly fs/1.18690!/menu/main/topColu mns/topLeftColumn/pdf/527033a.pdf

IM'NOVATION. https://www.imnovation-hub.com/society/types-of-smartsensors-applied-to-agriculture/\#0

LEE, Kai-Fu. Inteligência Artificial. Rio de Janeiro: Globo Livros, 2019.

LEE, Kai-Fu. 'The Algorithm Will See You Now'. AISuperpowers. 2019. https://aisuperpowers.com/blog/kai-fu-lee

LOHR, S. From agriculture to art - the AI wave sweeps. New York Times, Business Day. 2019. https://www.nytimes.com/2018/10/21/business/from-agriculture-to-artthe-ai-wave-sweeps-in.html

LYNCH, Shana. Andrew Ng: Why AI Is the New Electricity. Stanford Business. 2017. https://www.gsb.stanford.edu/insights/andrew-ng-whyai-new-electricity

MCCARTHY, J. What is artificial intelligence? Stanford University, 2007, p. 2-15.

MONTREAL. Montréal Declaration for a Responsible Development of Artificial Intelligence. 2020. https://www.montrealdeclarationresponsibleai.com/

NAGLER, J., VAN DEN HOVEN, J. \& HELBING, D. in Towards Digital Enlightenment, Springer International Publishing, 2019, p. 41-46. https://doi.org/10.1007/978-3-319-90869-4 5

O'CONNOR, Marry Catherine. How AI Could Smarten Up Our Water System. Medium. 2017. https://medium.com/s/ai-for-good/how-ai-could-smartenup-our-water-system-f965b87f355a

OECD. Science, Technology and Innovation Scoreboard 2017: the Digital Transformation. Organisation for Economic Co-operation and Development, Paris, 2017.

POLIDO, Fabrício Bertini Pasquot. Inteligência artificial entre estratégias nacionais e a corrida regulatória global: rotas analíticas para uma releitura internacionalista e comparada. Rev. Fac. Direito UFMG, Belo Horizonte,
n.
76,
pp.
229-256,
jan./jun.
2020. 
SANTOS DIVINO, Sthéfano Bruno. Reflexões sobre a inteligência artificial na agenda 2030 para o desenvolvimento sustentável. Revista Eletrônica Direito e Política, Programa de Pós-Graduação Stricto Sensu em Ciência Jurídica da UNIVALI, Itajaí, v.16, n.1, 10 quadrimestre de 2021. Disponível em: www.univali.br/direitoepolitica - ISSN 1980-7791.

https://www.direito.ufmg.br/revista/index.php/revista/article/view/2067/1 $\underline{931}$

RASP, Stephan; PRITCHARD, Michael S; GENTINE, Pierre. Deep learning to represent subgrid processes in climate models. PNAS. Vol. 115, n. 39, p. 9684-9689. https://doi.org/10.1073/pnas.1810286115. https://www.pnas.org/content/pnas/115/39/9684.full.pdf

SEARLE, J. Minds, brains, and programs. Behavioral and Brain Sciences. Vol. 3, n. 3. 1980, p. 417-424.

SÉVIGNY, B. Québec lays the groundwork for a world observatory on the social impacts of artificial intelligence and digital Technologies. 2018. http://www.scientifique-en-chef.gouv.qc.ca/en/nouvelles/quebecjette-bases-dun-observatoire-mondial-impacts-societaux-de-lintelligenceartificielle-numerique/

STARK Luke, PYLYSHYN, Zenon W. Artificial Intelligence (AI), cit.; 'Here's how Canada can be a global leader in ethical AI'. In: The Conversation. 2018. https://theconversation.com/heres-how-canada-can-be-a-globalleader-in-ethical-ai-90991

UNESCO. Artificial intelligence for sustainable development: challenges and opportunities for UNESCO's science and engineering programmes. 2019.

UNESCO. Canada first to adopt strategy for artificial intelligence. 2018. http://www.unesco.org/new/en/member-states/singleview/news/canada first to adopt strategy for artificial intelligence/

UNESCO. Towards a monopolization of research in artificial intelligence? Natural Sciences Sector. 2018. www.unesco.org/new/en/natural-sciences/sciencetechnology/single-viewsc-policy/news/towards_a_monopolization_of_research_in_artificial_ intellige

UNITED KINGDOM. AI Sector Deal One Year On. 2019. https://assets.publishing.service.gov.uk/government/uploads/system/uplo ads/attachment data/file/819331/AI Sector Deal One Year On Web. pdf

UNITED KINGDOM. Government response to House of Lords Artificial Intelligence Select Committee's Report on AI in the UK: Ready, Willing and Able?. 2018. https://www.parliament.uk/documents/lordscommittees/Artificial-Intelligence/AI-Government-Response2.pdf

VINUESA, R., AZIZPOUR, H., LEITE, I. et al. The role of artificial intelligence in achieving the Sustainable Development Goals. Nat Commun 11, 2020, p. 233. https://doi.org/10.1038/s41467-019-14108-y 
SANTOS DIVINO, Sthéfano Bruno. Reflexões sobre a inteligência artificial na agenda 2030 para o desenvolvimento sustentável. Revista Eletrônica Direito e Política, Programa de Pós-Graduação Stricto Sensu em Ciência Jurídica da UNIVALI, Itajaí, v.16, n.1, $1^{0}$ quadrimestre de 2021. Disponível em: www.univali.br/direitoepolitica - ISSN 1980-7791.

VIPKID. https://m.vipkid.com/

WWWF. Artificial Intelligence: the Road Ahead in Low and Middle-Income Countries. World Wide Web Foundation. https://webfoundation.org/docs/2017/07/AI Report WF.pdf

RECEBIDO EM: FEV/2020

APROVADO EM: AGO/2020 